\title{
Optimal Control of Plug-In Electric Vehicles Charging for Composition of Frequency Regulation Services
}

\author{
Roberto Germanà $(D)$ Francesco Liberati *(D), Emanuele De Santis ${ }^{(D)}$, Alessandro Giuseppi (D), \\ Francesco Delli Priscoli (D) and Alessandro Di Giorgio (D)
}

Department of Computer, Control and Management Engineering (DIAG), University of Rome "La Sapienza", 00185 Rome, Italy; germana@diag.uniroma1.it (R.G.); edesantis@diag.uniroma1.it (E.D.S.); giuseppi@diag.uniroma1.it (A.G.); dellipriscoli@diag.uniroma1.it (F.D.P.); digiorgio@diag.uniroma1.it (A.D.G.) * Correspondence: liberati@diag.uniroma1.it or liberatifnc@gmail.com; Tel.: +39-06-77274037

check for updates

Citation: Germanà, R.; Liberati, F.; De Santis, E.; Giuseppi, A.; Delli Priscoli, F.; Di Giorgio, A. Optimal Control of Plug-In Electric Vehicles Charging for Composition of Frequency Regulation Services. Energies 2021, 14, 7879. https:// doi.org/10.3390/en14237879

Academic Editors: Saeed Golestan and José Matas

Received: 14 September 2021 Accepted: 19 November 2021 Published: 24 November 2021

Publisher's Note: MDPI stays neutral with regard to jurisdictional claims in published maps and institutional affiliations.

Copyright: (c) 2021 by the authors. Licensee MDPI, Basel, Switzerland. This article is an open access article distributed under the terms and conditions of the Creative Commons Attribution (CC BY) license (https:/ / creativecommons.org/licenses/by/ $4.0 /)$.

\begin{abstract}
This paper presents a novel control system for the participation of plug-in electric vehicles (PEVs) in the provisioning of ancillary services for frequency regulation, in a way that is transparent to the driver and harmonized with the smart charging service requirements. Given a power-frequency droop curve, which specifies how the set of PEVs collectively participate to the provisioning of the frequency regulation service (we call this curve a "global" droop curve), we propose an algorithm to compute "local" droop curves (one for each PEV), which are optimized according to the current status of the PEV and the current progress of the smart recharging session. Once aggregated, the local droop curves match the global one (so that the PEVs contribute as expected to the provisioning of the ancillary service). One innovative aspect of the proposed algorithm is that it is specifically designed to be interoperable with the algorithms that control the PEV recharging process; hence, it is transparent to the PEV drivers. Simulation results are presented to validate the proposed solution.
\end{abstract}

Keywords: ancillary services; frequency regulation; plug-in electric vehicles

\section{Introduction}

In the recent years, the European energy market opened the possibility for third parties to provide regulation services (e.g., ancillary services). Among these control services, a very important role for the safe operation of the electricity network is given to the frequency control services, which aim at maintaining the network frequency in a safe range. Frequency regulation services are usually provided by traditional generators, through speed droop controllers that control the mechanical power applied to the rotor shafts based on their rotation speed. However, it is also possible to control the network frequency with other kind of generators, like wind farms [1,2] and photovoltaic plants [3]. Moreover, many papers intercepted the possibility to modulate the energy demand of heavy flexible electric loads, such as heating, ventilation, and air conditioning systems [4], and thermostatically controlled loads [5], in order to control network frequency. Among the flexible loads, PEVs emerged as good candidates for the provision of frequency regulation services, as their power consumption can be changed remotely by the charging stations, and the reaction time is very low. Anyway, the power absorbed by a single PEV is not enough to participate effectively to the ancillary service market, so it is fundamental to aggregate a set of PEVs and coordinate their action to provide the requested services. The possibility to aggregate many charging PEVs is becoming easier, due to the mass spreading of PEVs in the market and thanks to new high-speed/low-latency cellular communication means [6], which significantly reduce the cost to update the current charging point operator (CPO) infrastructure to provide frequency regulation services. Indeed, the authors of this paper proposed a novel architecture [7] that enables CPO charging stations to provide frequency regulation services over a $5 \mathrm{G}$ communication network, exploiting the $5 \mathrm{G}$ multi access edge computing [8] as edge computing node, without violating the time constraints to 
provide this kind of services. The proposed architecture [7], together with the results coming from this paper, will be implemented in the context of the European H2020 Project 5G-Solutions [9].

The problem of controlling PEVs for frequency regulation services has been studied in the literature by Yao, Wong, and Schober with focus on a robust control method to estimate the hourly regulation capacity (i.e., aggregated power margins) of the PEVs [10], or with a focus on the market bids for the frequency regulation services provided by PEVs [11]. Moreover, Xia et al. studied, in [12], the problem of computing power setpoints for fleets of PEVs in presence of fluctuating wind generators, using consensus.

In addition, the contribution of PEVs to fast frequency regulation has been studied in [13], where the frequency droop curves for each PEV cluster are computed using an improved harmony search algorithm, but without balancing the effort of each PEV based on its smart charging requirements.

Kuang et al. have studied in [14] the problem of providing frequency regulation by using dispersed PEVs, while maintaining the target state of charge (SOC) level requested by the PEV owners, but without changing dynamically over time the control droop curves to balance the effort among the different PEVs based on the drivers' requirements.

In [15], the authors provided a hierarchical control scheme to allocate frequency control effort among the charging PEVs that is able to ensure the final SOC level for the PEVs by real-time corrections of the allocated frequency control action. Anyway, this approach does not optimize the effort among the different PEVs, allocating it based on the frequency regulation capacity of each PEV.

Jia et al. have studied in [16] the problem of load frequency control using PEVs with inertia uncertainties and time-varying delays, and in particular focusing on the coordination among PEVs and power plants to provide such frequency control functionalities, but without considering the integration of smart charging system with load frequency control, which is one of the focuses of the presented paper.

In [17], the authors have discussed a demonstration of the provisioning of frequencycontrolled normal operation reserve (one of the primary frequency regulation services in the Nord Pool energy market) using three PEVs from different manufacturers. Differently from the present work, the charging setpoints to implement the frequency regulation service are computed by a central controller, which results in higher response times compared to the decentralized solution we present.

In [18], a model predictive control scheme to schedule the bidding in frequency regulation market is proposed, with the aim of maximizing the payment to PEV aggregators, based on a prediction of the frequency regulation market price built using a seasonalautoregressive integral-moving-average model. Though taking into account the current PEVs SOC level, the EV power schedule is limited to three possible states and, again, the effort is not balanced among PEVs; moreover the algorithm does not provide droop curves to be applied by the PEVs in response to frequency deviations.

Finally, in [19], Islam et al. propose a Markov decision process formulation of the problem of aggregating flexibility from the PEVs for providing frequency regulation services. The goal is to maximize the revenue of the aggregator. A possible limitation of this approach is in its scalability, and in the possibility of having a fine-grained control for each PEV (due to the use of the state aggregation technique).

The distinctive features of the present paper are: (i) we propose an approach in which frequency control actions are taken at each charging station based on local droop curves, which allows for very fast intervention, as required for providing fast frequency regulation. The parameters of the local droop curves are periodically updated by a central controller, which allows to equally balance the frequency control effort among the PEVs, while having a highly scalable scheme, since the central optimization problem is a linear one and is executed within larger time constraints with respect to the frequency regulation control loop ones; (ii) the droop curves are computed as to balance the control effort among the various PEVs, taking into account the smart charging requirements of the different 
users, on available dwelling time, current SOC, and final desired SOC; (iii) the presented algorithm exploits the availability of edge computing nodes, such as $5 \mathrm{G}$ multi-access edge computing, to reduce, as much as possible, communication latencies with the charging stations, and with the frequency meter.

In summary, the proposed approach is scalable, it allows for very fast frequency control action, and it ensures a correct balancing of the control action among the participating PEVs, by taking into account their individual conditions. Therefore, the algorithm summarizes the strengths found separately in the different works in literature.

The rest of the paper is structured as follows: Section 2 presents the reference system architecture and the interaction between traditional smart charging services and frequency regulation services; Section 3 presents the problem formulation, while Section 4 the proposed algorithm to compute the local droop curves for each charging PEV; Section 5 reports numerical simulation to validate the proposed algorithm.

\section{Reference Scenario and Problem Description}

In recent years, the European energy market has moved towards a separation between the roles of balance responsible party (i.e., the entities which are responsible for electricity network imbalances), and balance service provider (i.e., the entities which provide balancing services for the electricity network). In this context, the distribution system operators (DSOs) can rely on the balance service providers for their balancing services [20]. The CPO can, thus, take advantage of this market separation, becoming itself a balance service provider and providing ancillary services to the power network, by leveraging the possibility of changing the power setpoint of the charging station in real-time. Anyway, the CPO cannot participate to the ancillary service market with each separate charging station, since the power provided by each charging station is not in line with the power requirements to access to this market; instead, it can participate after aggregating many (even physically dispersed) charging stations, and so providing balancing services both at DSO and transmission system operator level. In this context, the authors of this paper proposed a smart charging solution that can enable fleets of charging PEVs to demand side management services [21].

Within the same scenario, it is possible, in principle, to provide also frequency regulation services, by leveraging the power margins provided by the smart charging system. Indeed, most of the time the charging PEVs charge with a power setpoint that is between zero and the maximum charging power, and so there are power margins both to increase and to reduce the power adsorbed by the PEVs. These margins can be aggregated and used to participate to the frequency regulation services.

Figure 1 shows the reference scenario considered in this study, which is based on the same novel architecture proposed in [7]. A set of PEVs are connected to charging stations that are remotely monitored and controlled by the CPO backend. The charging power setpoints actuated by the charging stations are periodically (e.g., every minute in [21]) computed by smart charging algorithms hosted in the "smart charging module". The goal of the smart charging module is to control the PEV recharging in the load area, in such a way that the PEVs are recharged in compliance with the user preferences (e.g., time available for recharging and amount of energy to recharge), and in compliance with the technical constraints of the grid. Hence, the role of the smart charging module is to ensure safe (for the vehicle and the grid), efficient (e.g., taking into account battery degradation), and economical smart charging service to the users. For an example of a possible implementation of the smart charging module, and the associated smart charging algorithms, the reader is referred, e.g., to our previous work [22].

The role of the "local droop curves computing module" instead is related with the provisioning of frequency control services. The module hosts a control algorithm which builds frequency control services by leveraging the flexibility offered by the PEVs. The module periodically computes and sends to the charging stations one power-frequency droop curve for each PEV performing smart charging, and agreeing to participate in frequency regula- 
tion. The droop curves are frequency/power curves that, given the current value of the network frequency, specify the power deviation with respect to the smart charging setpoint, i.e., how much the charging power setpoint computed by the smart charging module for the specific vehicle should be increased or decreased at any time, depending on the current grid frequency value. In absence of the frequency regulation service, the charging station simply implements the power setpoint it receives periodically from the smart charging module. When the PEV also participates in the frequency regulation, the charging station adds or subtracts to the smart charging setpoint a delta of power that is given by the value of the local droop curve at the current value of the grid frequency (frequency measurement is communicated to the charging station via $5 \mathrm{G}$ ). To be able to modulate, in real time, the power setpoint, as required in this scheme, the charging station needs to be equipped with a power converter (as in direct current charging stations). As discussed in the following, the different droop curves are computed in such a way that their total, cumulative effect follows a desired power-frequency droop curve, as if the PEV fleet was a unique entity providing frequency regulation services.

The relation among the two modules introduced above will be fully characterized in a future work. We remark here that each local droop curve is computed on the basis of the specific parameters of the related charging session. The logic is that the contribution of each PEV to the frequency regulation should be tuned depending on the current progress of the charging session, and on the technical characteristics of the PEV. On the other hand, the implementation of the droop curves by the single PEVs means that a different setpoint is actuated by the PEV, compared to the one that was computed by the smart charging module. The effect of this perturbation is compensated by the fact that the smart charging module periodically recomputes the charging schedules (see, e.g., [22]), so that the new charging schedule is computed taking into account, and to compensate, the effect of any possible deviation from the previous schedule caused by the participation of the PEV to the frequency regulation service (and by any other disturbance affecting the charging process). The effect of the perturbation on the smart charging session caused by the participation of the PEV in the frequency regulation service is measured by the smart charging module by measuring the current state of charge of the PEV, before each re-calculation of the smart charging setpoint. This scheme ensures that the two services, i.e., the smart charging service, and the frequency regulation one, can coexist, in a way that is transparent for the user.

The present works focuses entirely on the discussion of the control algorithm hosted by the local droop curves computing module. The interaction of this module with the smart charging module will be fully analyzed in a future publication.

In order to enable the charging stations to provide frequency regulation services, the system should be able to take a frequency measure and actuate the proper power setpoint to the PEV (increasing or reducing it based on the frequency deviation) within a very limited time (in the order of $300 \mathrm{~ms}$, according to the Fast Reserve pilot project of TERNA [23]), as analyzed in [7]. It is important to notice that this very strict time constraint has to be guaranteed just for frequency regulation functions, while other smart-charging functionalities (e.g., the computation of nominal power setpoints) have much larger time constraints, that are typically in the order of minutes. Installing a frequency meter capable of the above-mentioned time requirements inside each charging station would imply very high costs. However, by leveraging the new cellular communication technologies (e.g., 5G), it is possible to install just one (or few, for redundancy) frequency meter in each load area, and then spread the information to the other charging stations with negligible delay, thus matching the strict time requirements for the service. 


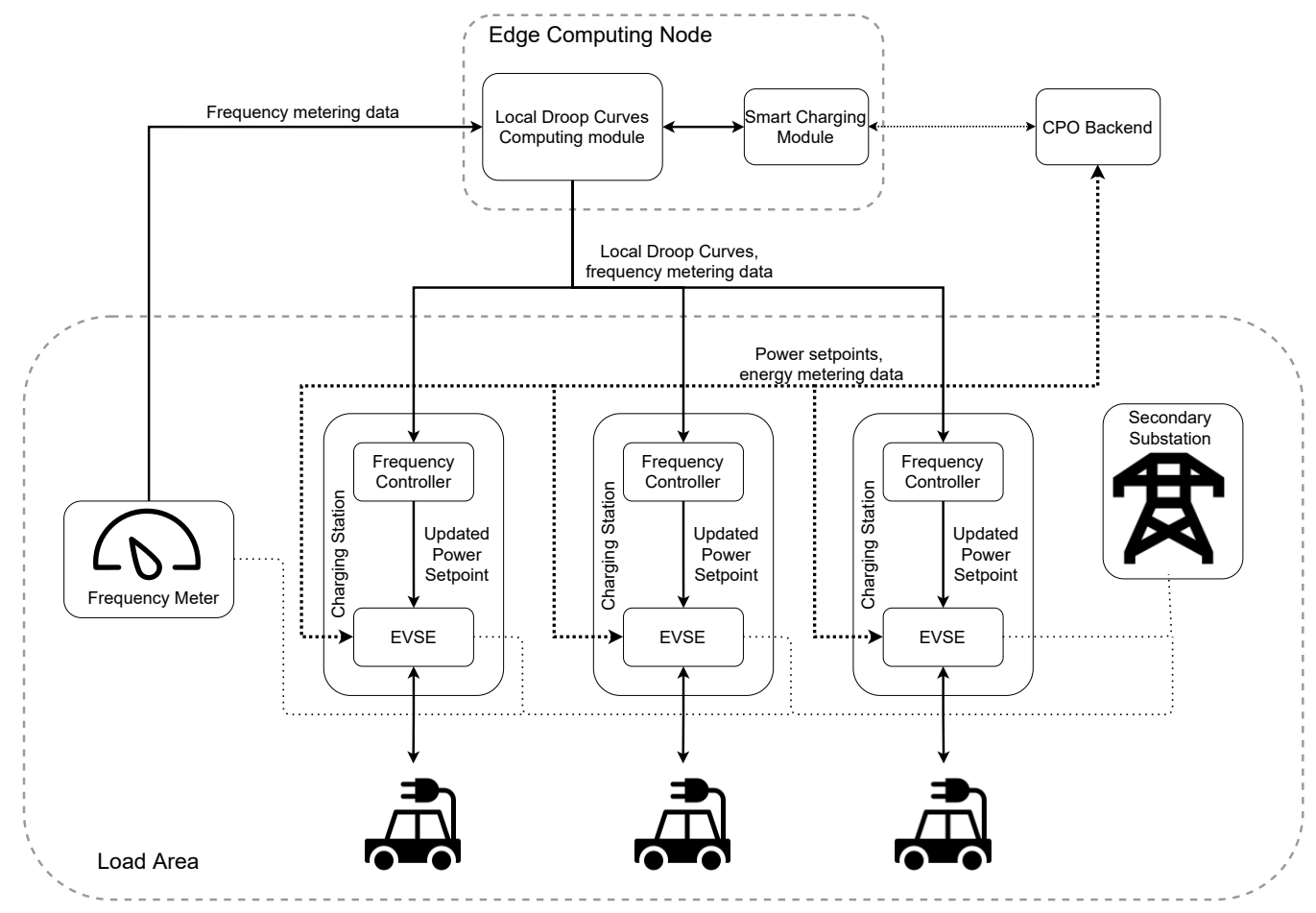

Figure 1. Reference System Architecture.

\section{Nomenclature and Problem Formulation}

In this section, we detail the proposed control algorithm hosted in the "local droop curves computing module", which periodically computes the local droop curves (i.e., one for each charging station), based on the current status of the ongoing charging sessions. In particular the local droop curves are computed with the same sampling time of the smart charging signals (e.g., nominal power setpoints for the charging sessions), so in the order of minutes. First of all, the used nomenclature is introduced.

Let $N$ be the number of PEVs connected at the generic time $k$ in the load area. The generic $n$-th PEV at time $k$ is characterized by:

- $\quad$ The current charging power level $P_{n, k}$;

- The maximum and the minimum possible charging power levels, respectively $P_{n}^{\max }>0$ and $P_{n}^{\min }$ (if $P_{n}^{\min }<0$, the PEV is enabled to discharging);

- $\quad$ The current SOC level $x_{n, k}$;

- The time left until the end of the charging session, $d_{n, k}>0$;

- The error, $e_{n, k}$, between the desired SOC, $x_{n}^{r e f}$, and the current one, $x_{n, k}$, i.e., $e_{n, k}:=$ $x_{n}^{r e f}-x_{n, k}$;

- The power deviation, $\Delta P_{n, k}$, at time $k$, for the n-th PEV, due to the participation in the frequency regulation service. This value is computed from a droop curve.

The proposed algorithm is aimed at optimally coordinating the connected PEVs in the participation to the provisioning of ancillary services. In more detail, the problem we tackle is that of optimally define $p-f$ droop curves at single PEV level (which we call local droop curves ), in such a way that, once combined, they match a given, desired droop curve (the global droop curve). The global droop curve defines how, collectively, the connected PEVs should react to frequency mismatches, as if they formed a unique entity participating in the provisioning of the ancillary service.

Figure 2 displays a general prototype of a global p-f curve. Focusing of the righthalf plane, the parameter $\Delta f_{\min }$ defines the deadband: the power variation is zero if the deviation of the frequency with respect to the reference frequency value is in the interval $\left[0, \Delta f_{\min }\right] . \Delta f_{\max }$ defines the frequency deviation limit after which the power variation 
saturates. $m^{\text {global }}$ defines the droop, i.e., the ratio between the variation of the power and the frequency deviation.

The algorithm presented next recovers the global droop curve as the sum of $N$ local droop curves. A notable aspect is that the design of each local droop curve takes into account the current status of the PEV's recharging process, as explained next.

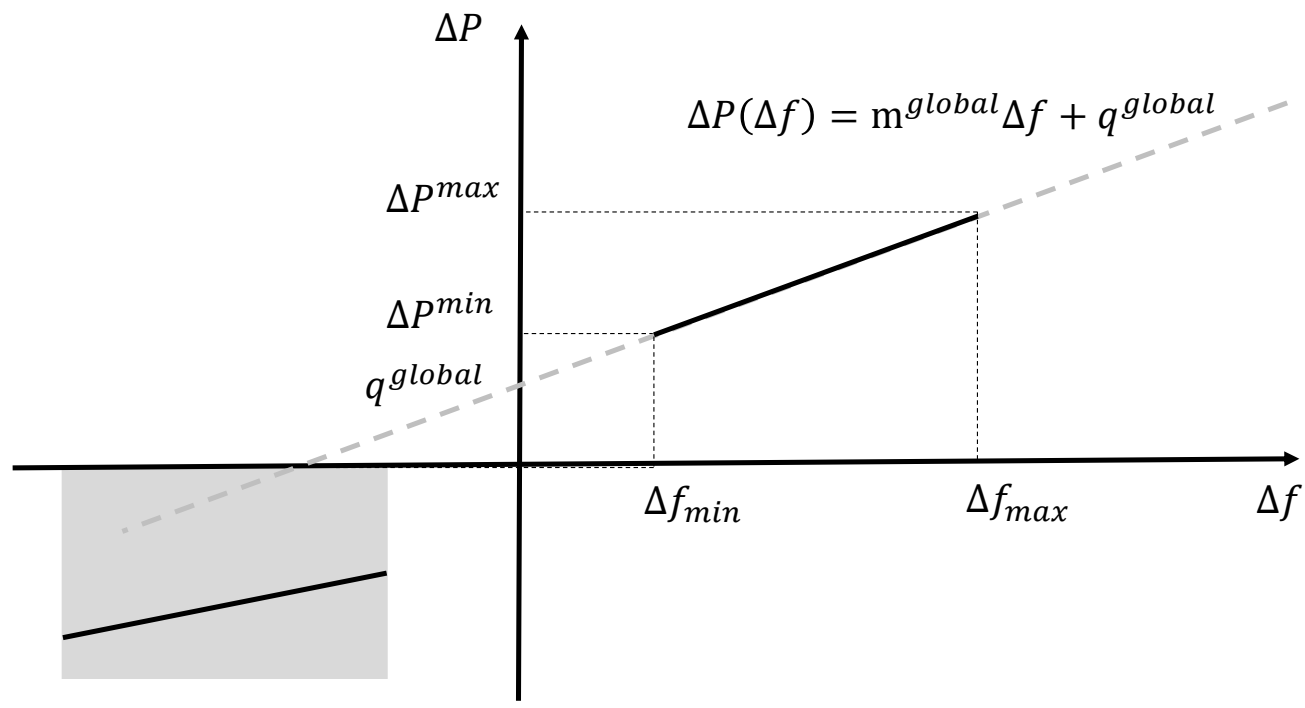

Figure 2. Global droop curve, with associated relevant parameters.

For the design of the local droop curves, we make the following assumption.

Assumption 1 (Shape of local droop curves). Local droop curves have a positive slope, and they are linear between $\Delta f_{\min }$ and $\Delta f_{\max }$.

Assumption 1 is included because it is in line with the common design principle of standard $\mathrm{p}-\mathrm{f}$ droop curves (the algorithm proposed in this paper could work also with non-linear droop curves). As implied by Assumption 1, the focus of this paper is on linear droop curves, i.e., on curves described by the equation:

$$
\Delta P_{n, k}(\Delta f)=m_{n, k} \Delta f+q_{n, k} .
$$

We focus on linear local droop curves because standard droop curves are linear, and because working with such type of functions results in a linear optimization problem, which has low computational complexity.

The algorithm proposed in this paper optimally designs each local droop curve by selecting the parameters $m_{n, k}$ and $q_{n, k}$, which are, therefore, the optimization variables of the problem. The algorithm is presented in the next section. To keep the description of the algorithm concise, and without loss of generality, we focus our attention on the design of the portion of the droop curves lying on the right half of the $\Delta f / \Delta P$ plane (see Figures 2 and 3 ). The part of the curve on the left half of the plane is designed in a similar way. 


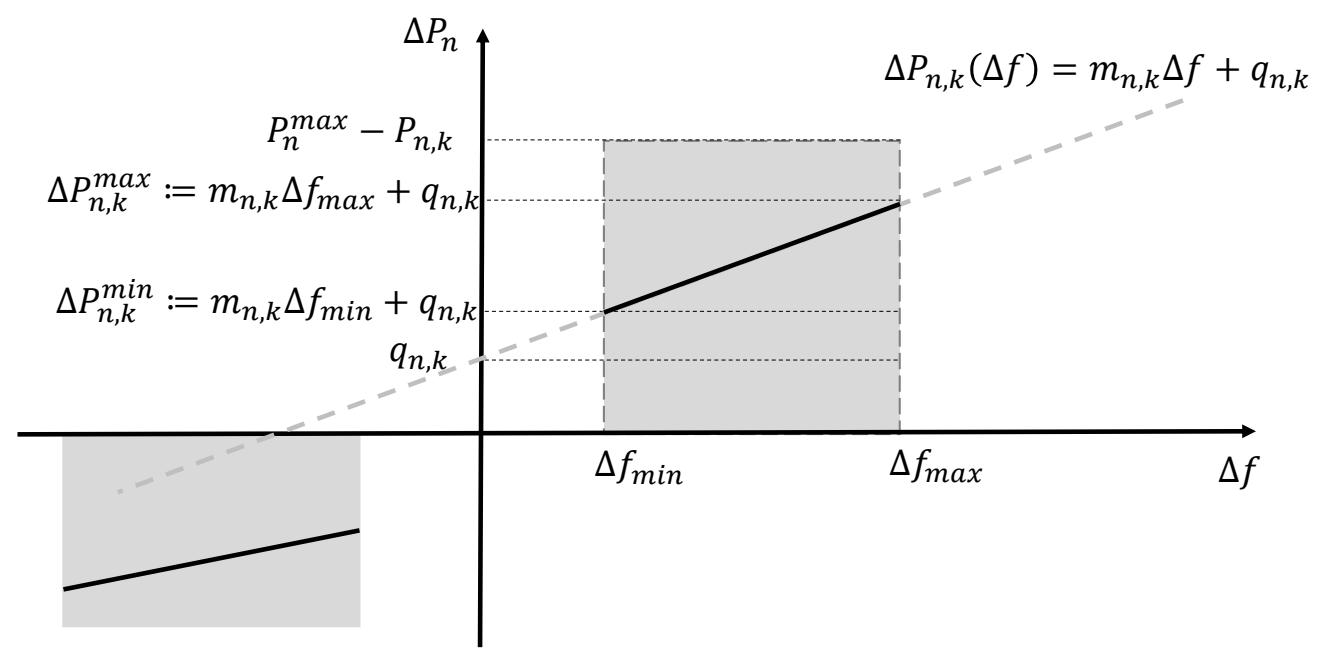

Figure 3. Local droop curve, with associated relevant parameters.

\section{Proposed Local Droop Curves Design Algorithm}

The design of the generic local droop curve (1) must respect constraints related to the shape of the local curve, and others related to the shape of the global droop curve. It must also respect certain limitations imposed by the current status of the charging sessions, as explained next.

\subsection{Local Droop Curve Design Constraints}

Figure 3 displays in gray the "design space" in which the generic n-th local droop curve can be drown.

The following constraints for each local curve are included.

$$
\begin{aligned}
& 0 \leq \Delta P_{n, k}^{\min }:=m_{n, k} \Delta f_{\min }+q_{n, k} \leq P_{n}^{\max }-P_{n, k}, \\
& 0 \leq \Delta P_{n, k}^{\max }:=m_{n, k} \Delta f_{\max }+q_{n, k} \leq P_{n}^{\max }-P_{n, k} .
\end{aligned}
$$

The above two constraints state that, respectively, at $\Delta f_{\min }$ and $\Delta f_{\max }$, the power increase for the single PEV must be between zero (i.e., corresponding to a null contribution to the frequency regulation services, and, therefore, having no impact on the current charging power level), and the maximum possible increase in charging power, i.e., $P_{n}^{\max }-$ $P_{n, k}$, which takes into account the current charging level, $P_{n, k}$, and the maximum possible one, $P_{n}^{\max }$. Notice however that (3) alone is not sufficient, since the maximum power increase might be affected also by the current SOC. For example, the maximum power increase for a vehicle that is fully charged is zero. For this reason, the following constraint is added:

$$
P_{n, k}+\Delta P_{n, k}^{\max } \leq \frac{x_{n}^{\max }-x_{n, k}}{T} .
$$

Equation (4) states that the maximum power increment is limited by the maximum energy that can be charged into the battery in the unit of time. $T$ is the sampling time of the algorithms, i.e., every $T$ seconds the local droop curves are re-computed.

Given the focus on linear droop curves, (2)-(4) ensure that the contribution of the single PEV is always feasible, also considering the current status of the charging session.

Next, in line with Assumption 1, local droop curves must have a positive slope, i.e.,

$$
m_{n, k} \geq 0 .
$$

This, of course, also implies that $\Delta P_{n, k}^{\min } \leq \Delta P_{n, k}^{\max }$. 


\subsection{Global Droop Curve Design Constraints}

The $\mathrm{p}-\mathrm{f}$ droop curve arising from the superimposition of the local droop curves can be written as

$$
\Delta P(\Delta f)=m_{k} \Delta f+q_{k}
$$

where

$$
m_{k}=\sum_{n=1}^{N} m_{n, k}, \quad q_{k}=\sum_{n=1}^{N} q_{n, k} .
$$

The following additional constrains are included to ensure that the superimposition of the local droop curves matches the desired global droop curve:

$$
\begin{gathered}
m_{k}=m^{\text {global }}, \\
q_{k}=q^{\text {global }} .
\end{gathered}
$$

\subsection{Target Function}

In the above subsections, we have presented the constraints that must be respected in the selection of the parameters $m_{n, k}$ and $q_{n, k}$, in order for the deriving local curves and global curve to be feasible d-f droop curves. In the following, we present the formulation of the proposed target function, to ensure that the parameters $m_{n, k}$ and $q_{n, k}$ are selected in an optimal way, according to the current status of the charging session of the PEVs participating in the provisioning of the ancillary service. The proposed target function to be minimized is:

$J_{k}=\sum_{n=1}^{N}-\alpha_{1} e_{k}\left(\Delta P_{n, k}^{\min }+\Delta P_{n, k}^{\max }\right)+\alpha_{2} d_{k}\left(\Delta P_{n, k}^{\min }+\Delta P_{n, k}^{\max }\right)+\alpha_{3} m_{k}^{\max }+\alpha_{4} \Delta P_{k}^{\max _{r}}+\alpha_{5} \Delta P_{k}^{\min _{r}}$,

where $\alpha_{1}, \ldots, \alpha_{5} \in[0,1]$ are such that $\sum_{i=1}^{5} \alpha_{i}=1$, and $m_{k}^{\max }, \Delta P_{k}^{\max _{r}}, \Delta P_{k}^{\min _{r}}$, are auxiliary variables such that:

$$
\begin{gathered}
m_{n, k} \leq m_{k}^{\max }, \quad m_{k}^{\max } \geq 0, \\
\frac{\Delta P_{n, k}^{\max }}{P_{n}^{\max }-P_{n, k}} \leq \Delta P_{k}^{\max _{r}} \quad \forall n \in N, \\
\frac{\Delta P_{n, k}^{\min }}{P_{n}^{\max }-P_{n, k}} \leq \Delta P_{k}^{\min _{r}} \quad \forall n \in N .
\end{gathered}
$$

Coefficients $\alpha_{1}, \ldots, \alpha_{5}$ can be used to weight the terms of the objective function. It is easy to see from (10) and (11) that, at the optimum, $m_{k}^{\max }$ is equal to the maximum value of $m_{n, k}$, for $i=1, \ldots, N$. Hence, the inclusion of this term in (10) has the goal of minimizing the maximum value of $m_{n, k}$, i.e., of balancing the effort of the participation in the ancillary service provisioning among the PEVs, aiming to avoid that some vehicles are assigned steep droop curves (i.e., high values of $m_{n, k}$ ). The last two terms in (10), similarly to $m_{n, k}$, contribute to spread the effort between the vehicles, by minimizing the maximum share of the available power margin that each vehicle contributes to frequency regulation (see (12) and (13)).

The first and the second terms in (10) instead are included to take into account also the current status of the charging session of each PEV, and, specifically, to give priority to the PEVs with a larger SOC error (the first term-notice the minus sign), and the ones with a smaller remaining charging time (the second term). The local droop curves associated to these PEVs, will have more pronounced slope and /or a higher power at $\Delta f_{\min }$. As a result, they will contribute more to the provisioning of the ancillary service, which will help them in reaching earlier the desired SOC level. Notice that, when the SOC error $e_{k}$ is negative (i.e., the current SOC is higher than the reference), then the PEV will contribute less to the provisioning of the service, which is a consistent behavior. Finally, recall that, for the sake of brevity, we focused in the paper in the design of the right-half part of the 
droop curves. The design of the left-half part of the curve, also in terms of the choice of the target function, can be carried out with similar considerations.

\section{Numerical Tests}

Simulations have been performed in Julia [24], version 1.6.0, on an standard computer (3.3 GHz, I7 processor with 16 GB RAM). The simulation scenario is as follows. We consider a charging load area that participates to the frequency regulation service. We assume that the charging sessions active in the load area are enough to provide the required power-frequency curve, i.e., that the composition algorithm presented in Section 4 admits a solution.

In the following, two simulation scenarios are discussed, to validated the proposed approach:

1. Scenario 1: we run the algorithm in a balanced scenario, i.e., considering a set of charging sessions that are homogeneous in terms of power margin flexibility, SOC error, and charging time availability;

2. Scenario 2: we run the algorithm in a scenario in which the charging sessions have different power margins, different SOC errors, and time flexibility.

The two scenarios are meant to show that, from one side, the algorithm is able to come up with a fair and balanced assignment of local droop curves among the participating PEVs while, on the other hand, taking always in consideration the real time SOC and time flexibility status of the participating PEVs, as determined by the respective charging session status, controlled by smart charging algorithms.

\subsection{Scenario 1: Local Droop Curves Assignment in a Balanced Scenario}

The first set of simulations is aimed to show how the algorithm is able to design local droop curves that result in a balanced distribution of the regulation effort, among the participating PEVs. To this end, we consider 3 active charging sessions, characterized by the same dwelling time and SOC error. The distinguishing attribute between the sessions is the available margin of power, $\Delta P_{n, k}^{\max }$, which reflects:

- The possible presence of different charging technologies in the load area, i.e., the fact that the charging sessions are characterized in general by different maximum power, depending on the charging technology;

- The presence of smart charging sessions, i.e., the fact that the charging sessions happen at different charging levels, which are in general different from the maximum possible charging level.

Indeed, the algorithm must be able to work in presence of smart charging sessions ongoing at different charging levels.

The first simulation in this scenario shows the case in which the charging sessions are characterized by the same power margin. Specifically, the charging sessions are characterized by a maximum charging power of $150 \mathrm{~kW}$, and by a common charging setpoint of $100 \mathrm{~kW}$, resulting in a power margin of $50 \mathrm{~kW}$. The global droop curve, for over frequency events, is characterized by a maximum power deviation of $105 \mathrm{~kW}(70 \%$ of the available power margin of $150 \mathrm{~kW}$ ), and by a frequency range $\left[\Delta f_{\min }, \Delta f_{\max }\right]=[500,1500] \mathrm{mHz}$. In correspondence with the minimum of the bandwidth, the given global droop curve is characterized by a power deviation of $10 \mathrm{~kW}$. Figure 4 shows the result of the algorithm. 


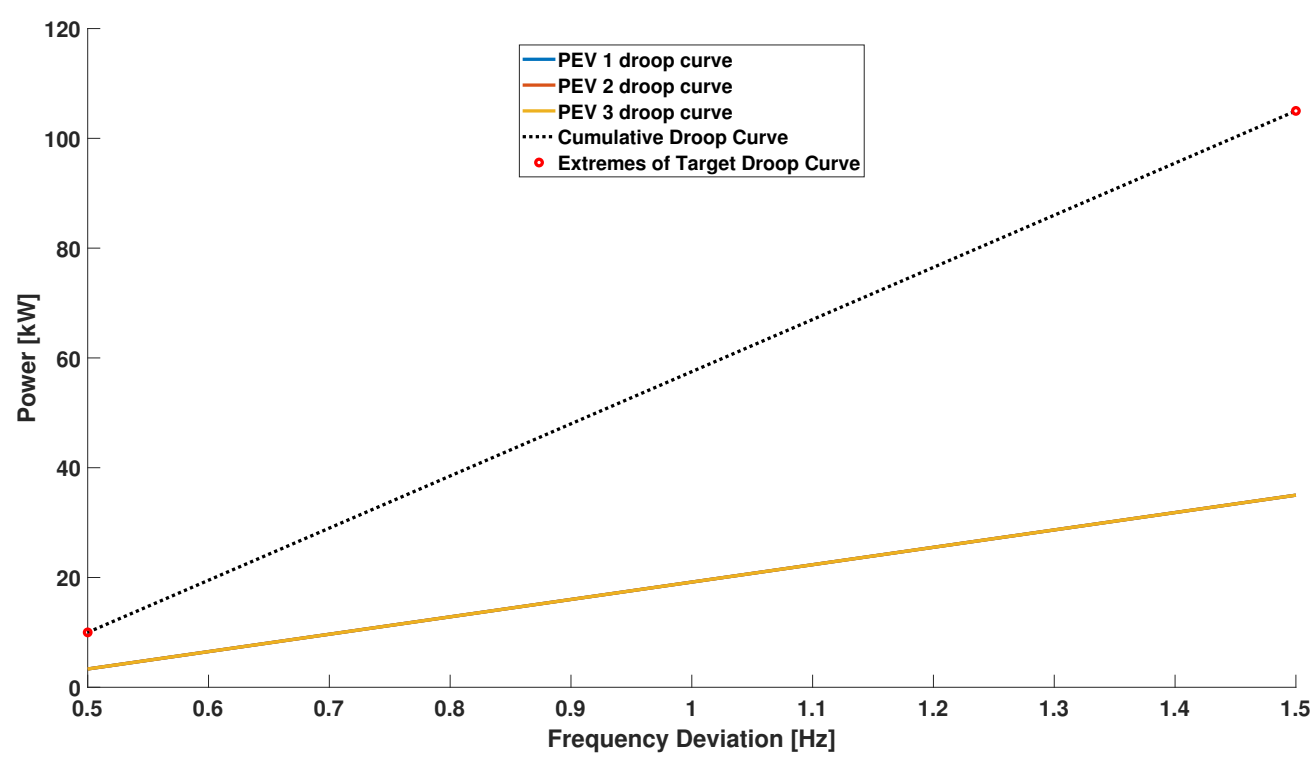

Figure 4. Scenario 1, balanced conditions: resulting local and global droop curves.

The algorithm determines the optimal distribution as an equal allocation of droop curves between the charging session. The target droop curve is identified in the plot with the red-dashed line, instead the resultant droop curve is represented by the black-dotted line. Figure 4 shows that the sum of the local curves matches exactly the target global one. The presence of the last term in the cost function determines an exact balancing of the frequency regulation service among the charging sessions, i.e., leading to identical local droop curves. Figure 5 shows the percentage of maximum usage of the power margin of each vehicle, i.e., the ratio between the value of the local droop curve corresponding to the frequency deviation $\Delta f_{\max }$ and the available margin of power. This plot confirms what already discussed: the algorithm distributes the effort in order to assign the same maximum relative usage to each vehicle's power margin, in this case $70 \%$.

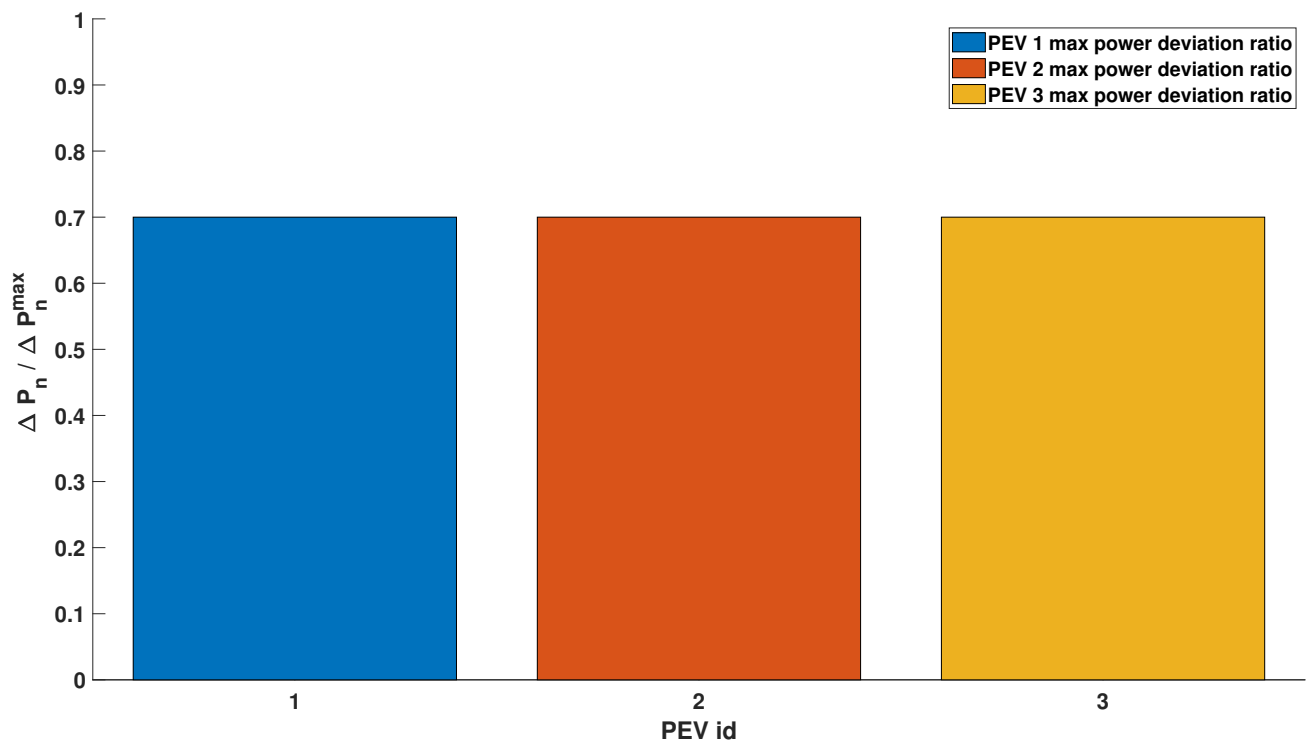

Figure 5. Scenario 1, balanced conditions: fraction of the maximum PEV power margin used for each PEV.

Typically, in smart charging, different charging power set-points are assigned to each charging session. As explained, this leads to different power margins, i.e., different levels of maximum contribution that could be provided by each PEV. This unbalance is considered by the proposed algorithm during the composition of the local droop curves. This is shown 
in the next simulation, which assumes three charging sessions characterized by different power margins, as summarized in Table 1.

Table 1. Charging sessions.

\begin{tabular}{ccccc}
\hline PEV ID & $\boldsymbol{P}_{\boldsymbol{n}, \boldsymbol{k}}[\mathrm{kW}]$ & $\boldsymbol{P}_{\boldsymbol{n}}^{\max }[\mathbf{k W}]$ & $\boldsymbol{e}_{\boldsymbol{k}}[\%]$ & $\boldsymbol{d}_{\boldsymbol{k}}[\%]$ \\
\hline 1 & 75 & 150 & 10 & 10 \\
2 & 50 & 100 & 10 & 10 \\
3 & 25 & 50 & 10 & 10 \\
\hline
\end{tabular}

The global droop curve that has to be composed is characterized by a maximum droop value of $75 \mathrm{~kW}$, i.e., $50 \%$ of the overall available margins. Note that all the charging sessions are performed at the $50 \%$ of maximum power, so they are characterized by different absolute power margins, $\Delta P_{n, k}$, but by the same percentage margin.

Figure 6 displays the droop curves assigned to each vehicle.

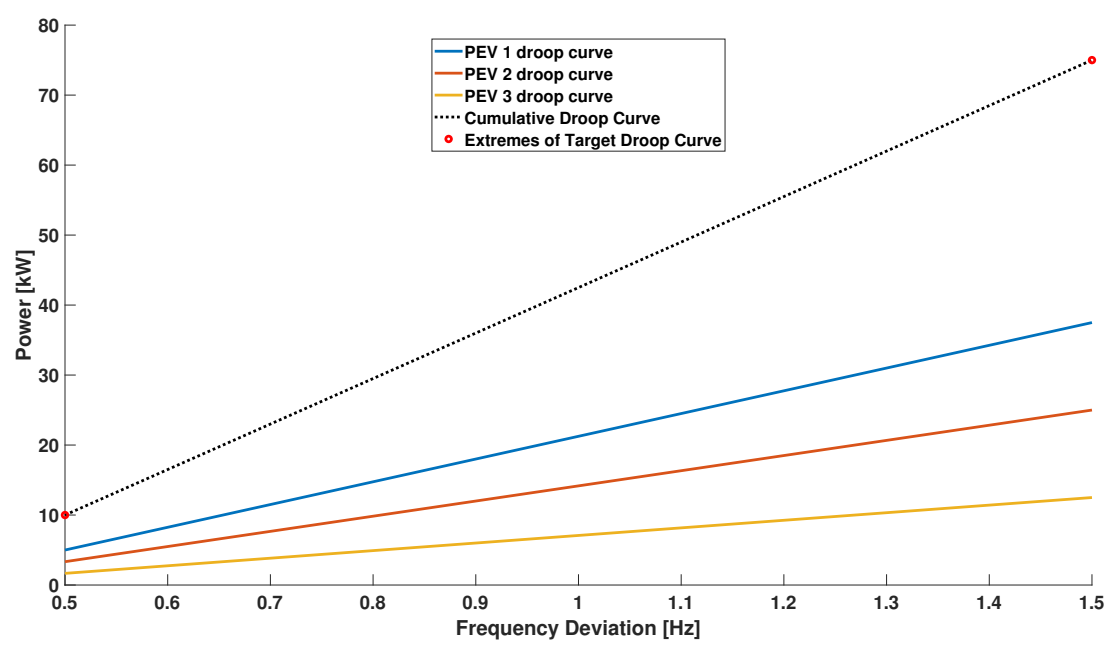

Figure 6. Scenario 1, different power margins: resulting local and global droop curves (request of $50 \%$ of the overall power margins).

The algorithm allocates the curves in order to distribute the relative effort equally between the sessions. Figure 7 highlights the equal distribution.
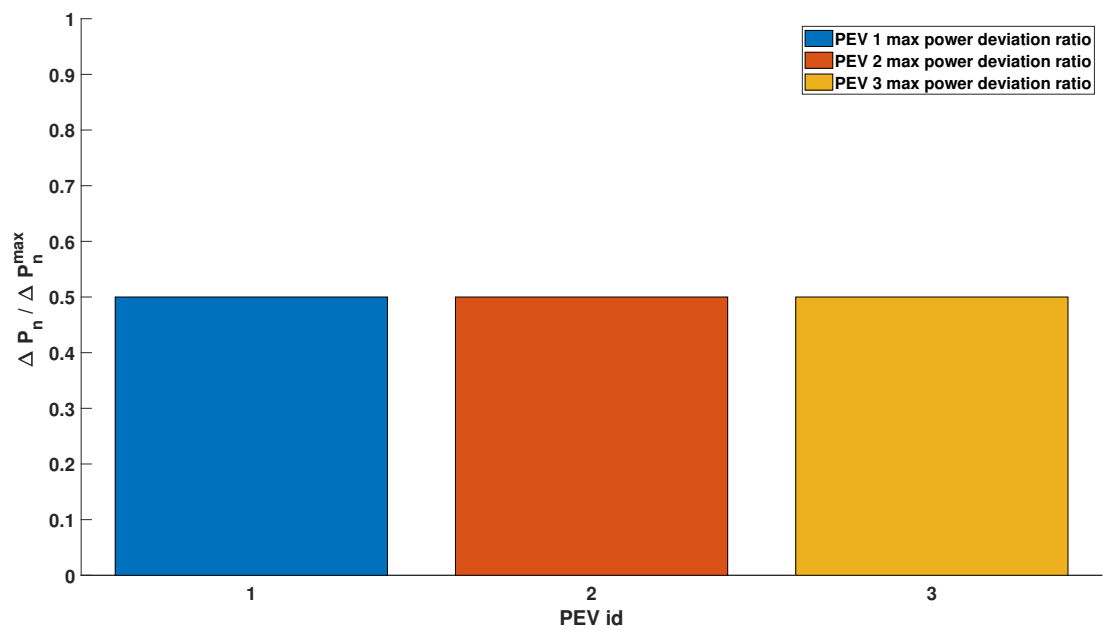

Figure 7. Scenario 1, different power margins: fraction of the maximum PEV power margin used for each PEV (request of $50 \%$ of the overall power margins). 
It is interesting to see the result of the same simulation, when the overall maximum power deviation increases. The same simulation is now performed with a maximum global droop curve value of $105 \mathrm{~kW}$, i.e., $70 \%$ of available margins. The increased request of power could be accomplished using the same strategy as before, so splitting the effort equally between the sessions. This strategy does not consider the fact that an equal distribution of power margins will impose a steeper droop curves to the sessions with larger margins, with a negative impact on the battery system. A steeper curve means a more aggressive response of the battery system to the frequency variations, with the consequently stress on power electronics and its effects on the battery temperature and cells health. The strategy presented in this work instead takes into account also the curve slope $m_{n, k}$ and the intercept $q_{n, k}$. As a result, the system distributes the additional effort needed to reach the new target droop curve less equally (Figure 8) but fair distributes the power electronics stress (Figure 9).

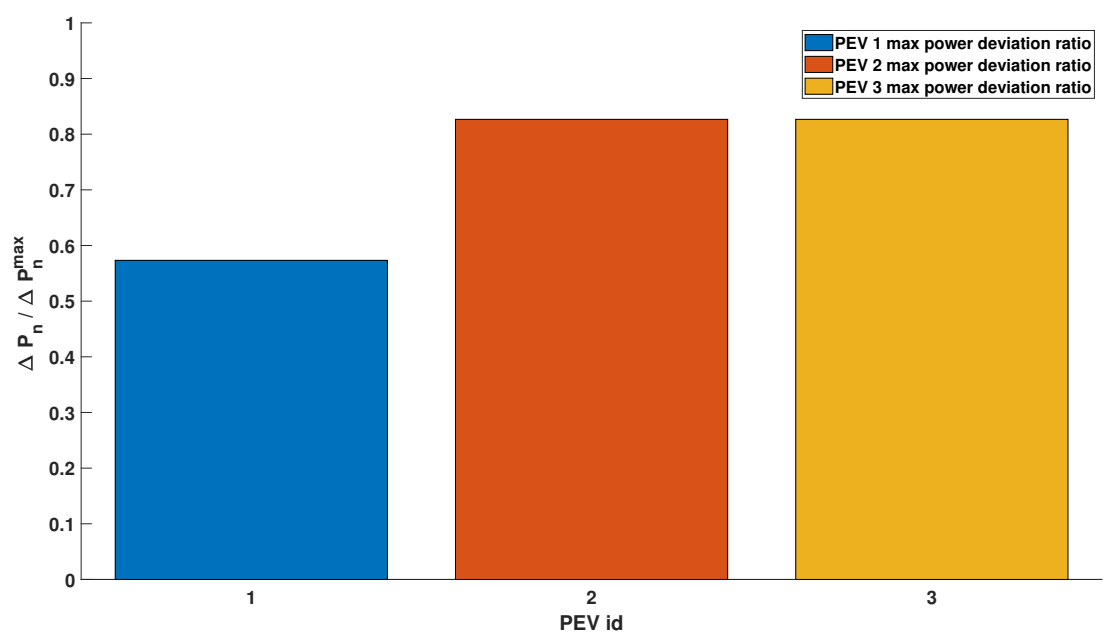

Figure 8. Scenario 1, different power margins: fraction of the maximum PEV power margin used for each PEV (request of $70 \%$ of the overall power margins).

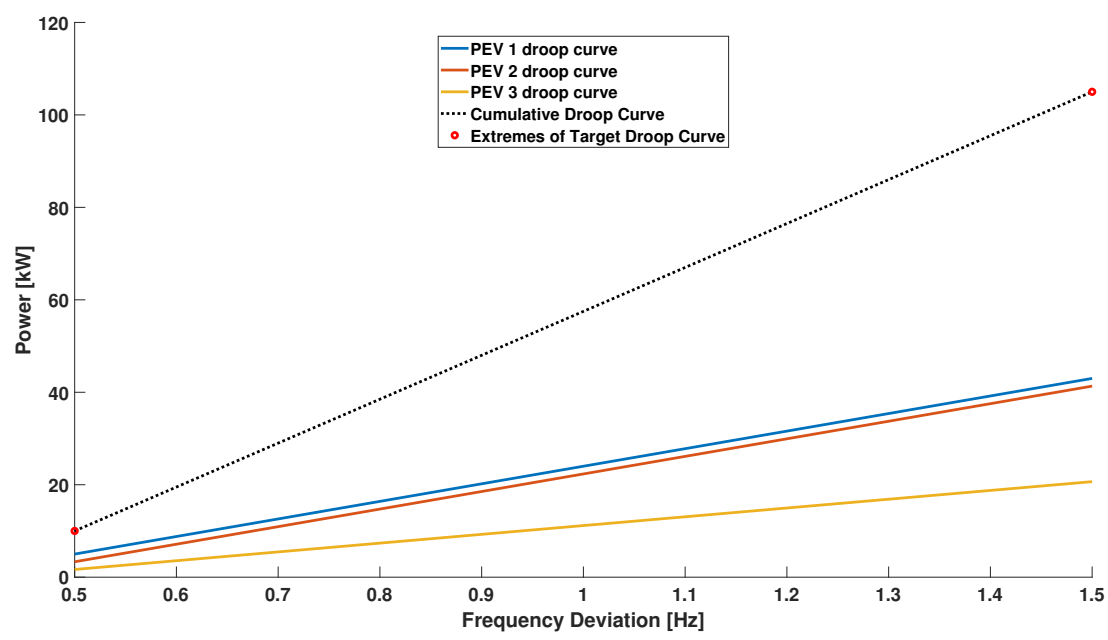

Figure 9. Scenario 1, different power margins: resulting local and global droop curves (request of $70 \%$ of the overall power margins).

\subsection{Scenario 2: Local Droop Curves Assignment in an Unbalanced Scenario}

The interconnection between the smart charging and the frequency regulation service is completed by considering the charging preferences expressed by the driver, i.e., the desired final state of charge and the charging time. Summarizing what already discussed about the target function (10), the algorithm gives a priority in the assignment of droop 
curves to the PEVs with a greater state of charge error and/or a shorter dwelling time (i.e., to those PEVs that will benefit from an increase in the charging rate).

The simulations presented below (Figures 10 and 11) will illustrate the capability of the designed algorithm to link the droop curve assignment with the charging status and the user preferences.

The balanced scenario is now re-proposed, but this time with different state of charge errors and dwelling times for the three PEVs. Table 2 summarizes the charging session status at the time when the proposed algorithm for allocating local droop curves is run.

PEV 1 is characterized by the greater SOC error, followed by PEV 2. The algorithm recognizes this condition and prioritizes the assignment of power to these two vehicles. The prioritization is in turn weighted by the respective SOC error.

Table 2. Charging sessions.

\begin{tabular}{ccccc}
\hline PEV ID & $\boldsymbol{P}_{\boldsymbol{n}, \boldsymbol{k}}[\mathrm{kW}]$ & $\boldsymbol{P}_{\boldsymbol{n}}^{\max }[\mathbf{k W}]$ & $\boldsymbol{e}_{\boldsymbol{k}}[\%]$ & $\boldsymbol{d}_{\boldsymbol{k}}[\%]$ \\
\hline 1 & 150 & 100 & 80 & 10 \\
2 & 150 & 100 & 40 & 10 \\
3 & 150 & 100 & 30 & 10 \\
\hline
\end{tabular}

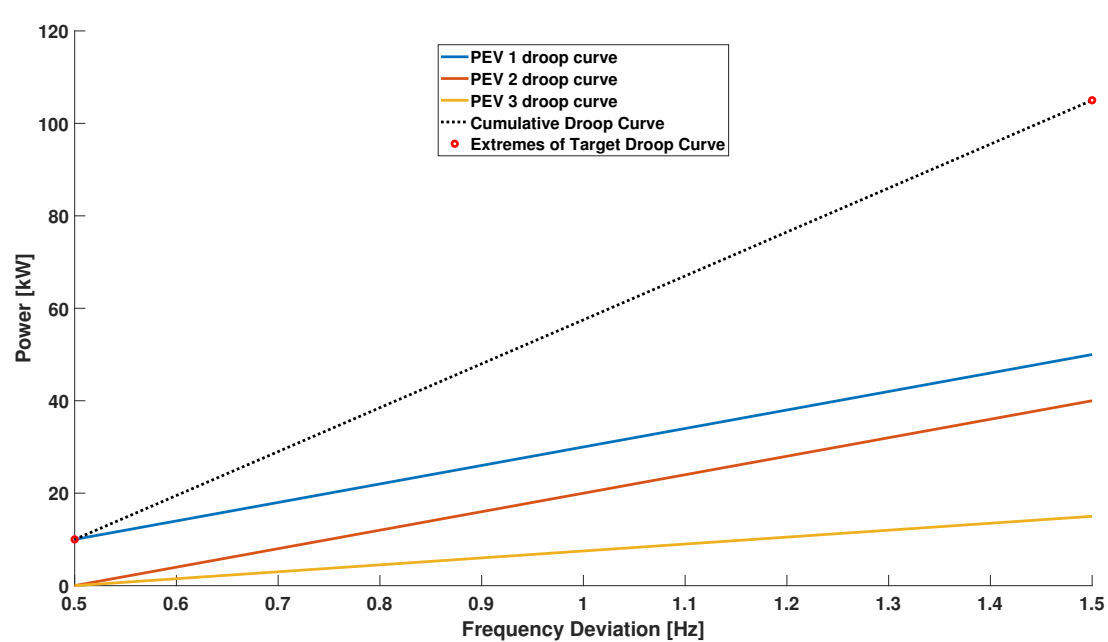

Figure 10. Scenario 2 balanced margins and unbalanced SOC errors: resulting local and global droop curves (request of $70 \%$ of the overall power margins).

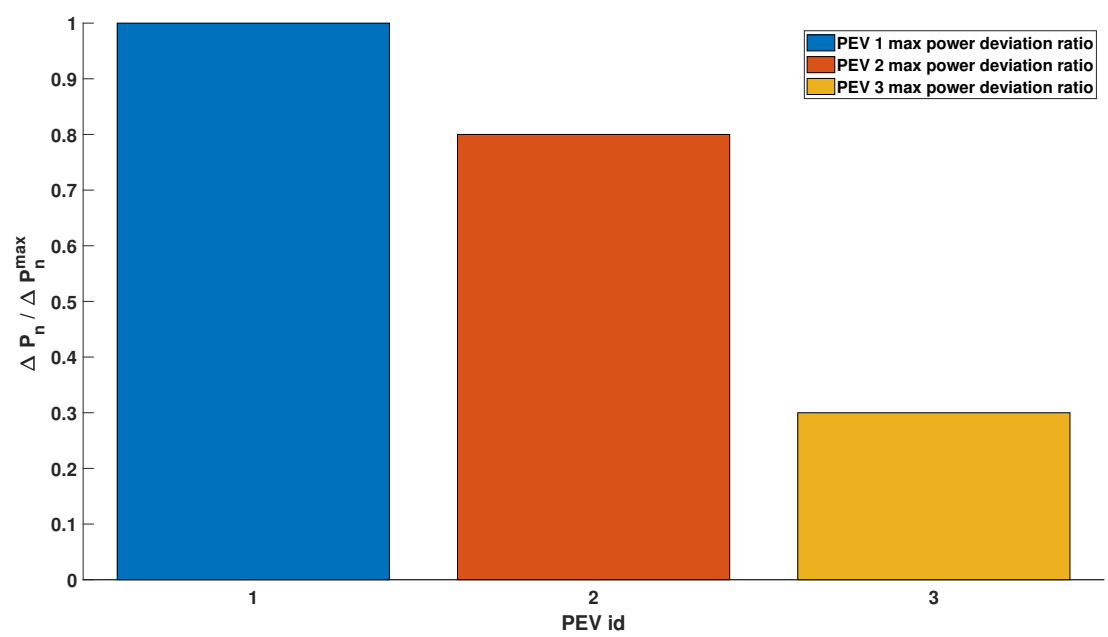

Figure 11. Scenario 2 balanced margins and unbalanced SOC errors: fraction of the maximum PEV power margin used for each PEV (request of $70 \%$ of the overall power margins). 
The SOC error is one of the two charging preferences taken into account in the droop curve assignment. In the above simulation, the dwelling time was the same for the three PEVs. Figures 12 and 13 show the case in which the PEVs 1 charging session, characterized by a SOC error of $80 \%$, is also characterized by a remaining dwelling time of $50 \%$. In this case, the SOC error prioritization is compensated by the available dwelling time, so the algorithm identifies as optimal strategy to equal distribute the effort between the vehicles.

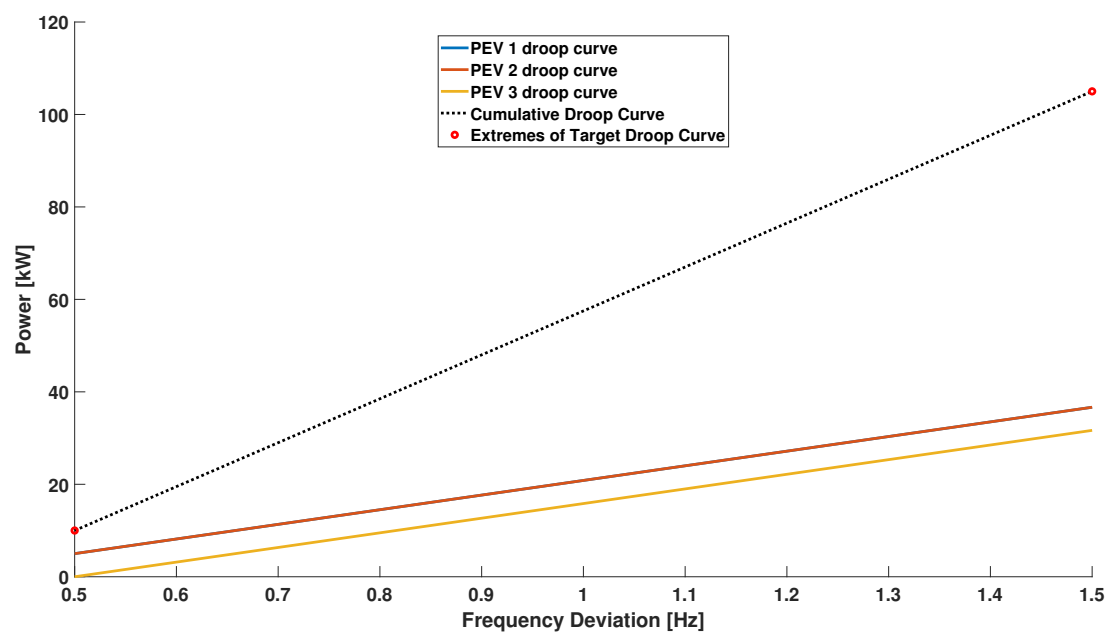

Figure 12. Scenario 2 balanced margins and unbalanced SOC errors and dwelling times: resulting local and global droop curves (request of $70 \%$ of the overall power margins).

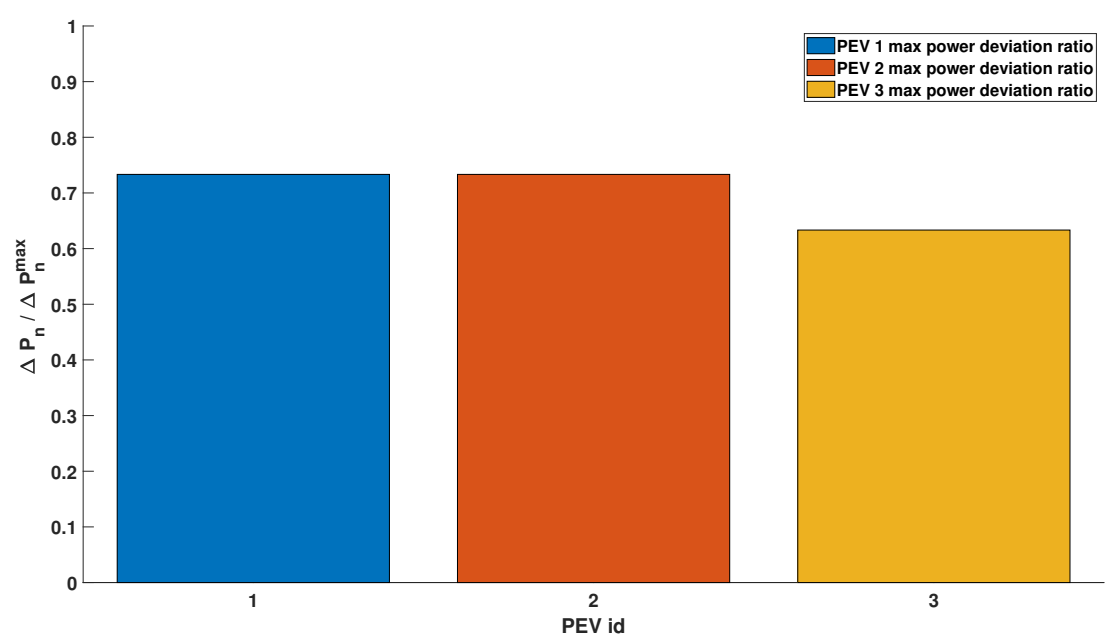

Figure 13. Scenario 2 balanced margins and unbalanced SOC errors and dwelling times: fraction of the maximum PEV power margin used for each PEV (request of $70 \%$ of the overall power margins).

\subsection{Notes on the Computational Complexity of the Algorithm}

The optimization problem associated to the proposed algorithm is linear, with continuous variables. This is the category of optimization problems that is the most consolidated in the literature, and for which efficient and well established optimization algorithms exist. The optimization problem was built in Julia and solved with Gurobi 9.1 [25].

Quadratic cost functions could be also used for this kind of problem, as they are an efficient choice in terms of effort distribution (e.g., the third term in the objective function could be replaced by the term $\alpha_{3} m_{n, k}^{2}$, and similarly the last two terms). However, quadratic problems are more complex, and require more computational resources than linear ones.

Considering that this algorithm is designed to work in conjunction with a smart charging system that updates the charging set points with a rate of minutes, and considering the amount of power needed for the participation to the frequency regulation services (e.g., in Italy, the Pilot Project fast reserve requires at least $5 \mathrm{MW}$ of aggregated power [23]), 
we have to face the issue of finding the solution of a large scale optimization problem in a short amount of time. For this reason, we decided to opt for a linear formulation. To test the scalability of the proposed algorithm, several simulation have been performed, considering scenarios of various dimension. Figure 14 reports the result of local droop curves computation in a scenario with 1000 PEVs participating in the frequency regulation. This simulation does not consider a specific charging technology, the maximum charging and discharging power rates for a generic charging session are randomly chosen, respectively, between $0 \mathrm{~kW}$ and $150 \mathrm{~kW}$, and $-150 \mathrm{~kW}$ and $0 \mathrm{~kW}$. Additionally, the user preferences are assigned randomly. The simulation shows how the proposed algorithm assigns different droop curves considering the current load area requirements ( $70 \%$ of the maximum available aggregated power margin), and the charging sessions status (the result is a uniform distribution of droop curves with no particular clustering). As already mentioned, being the problem linear with continuous variables, very low computational time was observed in various experiments, always between 1 and $1.3 \mathrm{~s}$.

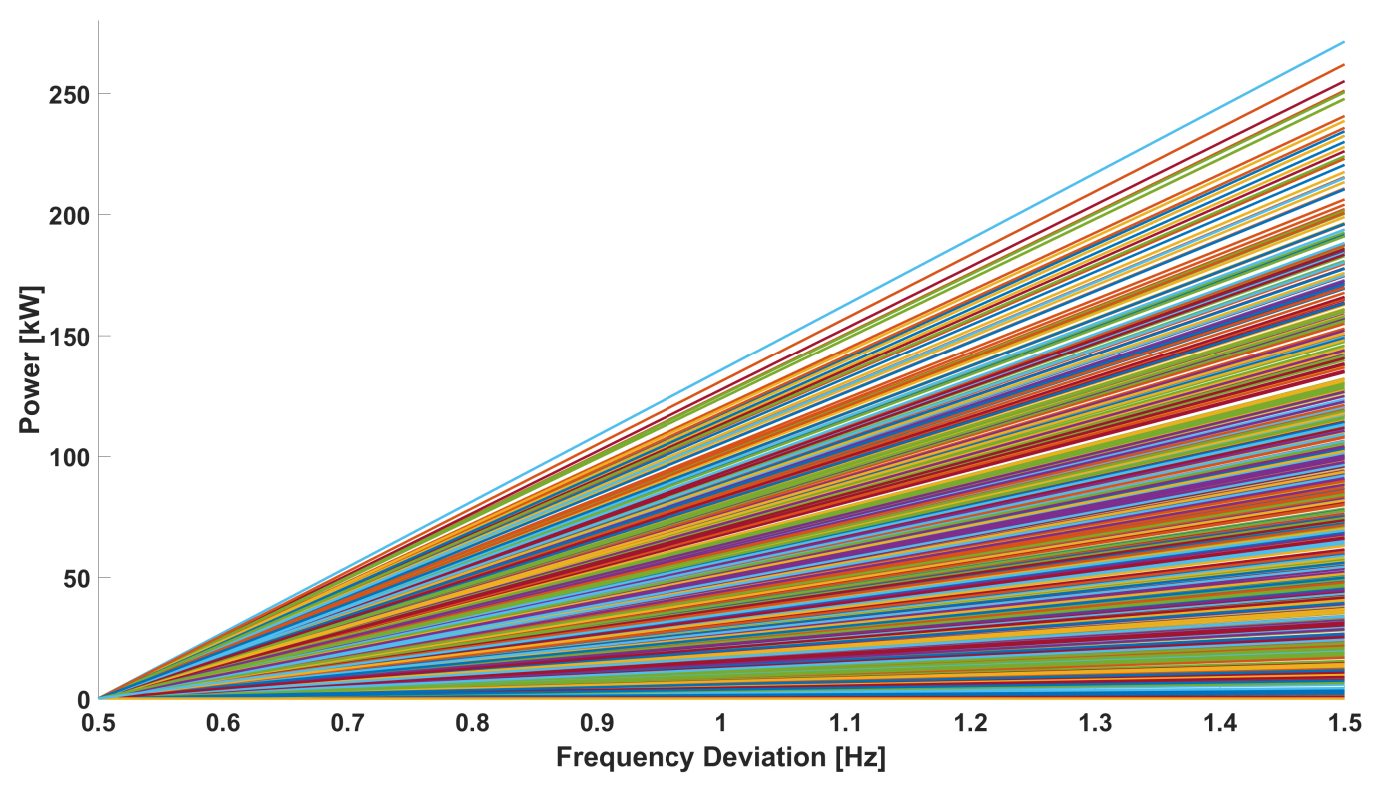

Figure 14. Simulation with 1000 PEVs.

Finally, the algorithm was tested with 100,000 contemporary charging sessions. The solution time was always between 30 and $50 \mathrm{~s}$, which is acceptable, in view of integrating the proposed algorithm with the smart charging algorithm.

\section{Conclusions}

This paper has presented a novel control algorithm for enabling the participation of smart charging plug-in electric vehicles (PEVs) to the provisioning of frequency regulation services. This has a positive value for the grid, providing an additional source of flexibility to ensure grid stability, and for the PEV drivers as well, providing to them an additional stream of revenues, which will lower the cost of ownership of the PEVs. The proposed algorithm computes local frequency-power droop curves, one for each active charging session. These curves specify how the charging power setpoint should be changed in real time, in response to frequency deviations from the nominal value. The local curves computation takes into account the real time status of the charging sessions (in terms of time left until the end of the charging session, current SOC, and energy left to charge), which is fundamental to ensure that the frequency regulation service is interdependent and harmonized with the smart charging service, and thus transparent to the PEV user. The superimposition of the local droop curves has to match a desired load area droop curve, which specify how the PEVs in the load area should collectively react to a frequency deviation. In this way, the aggregate of PEVs can provide frequency regulation services to 
the market. Numerical simulations have shown that the proposed algorithm is effective in assigning local droop curves in a fair way, which takes into account the different status of the charging sessions. Additionally, the algorithm scales well, and is able to cope with aggregates of tens of thousands of PEVs.

Author Contributions: Conceptualization, R.G., F.L., E.D.S. and A.D.G.; Formal analysis, F.L.; Investigation, R.G., F.L., E.D.S., A.G. and A.D.G.; Methodology, R.G., F.L. and A.D.G.; Project administration, F.D.P. and A.D.G.; Resources, F.D.P. and A.D.G.; Software, R.G.; Supervision, F.L., F.D.P. and A.D.G.; Validation, R.G., F.L., E.D.S., A.G. and A.D.G.; Visualization, R.G.; Writing-original draft, R.G., F.L. and E.D.S.; Writing-review and editing, F.L., A.G., F.D.P. and A.D.G. R.G. and F.L. are co-first authors of the paper. All authors have read and agreed to the published version of the manuscript.

Funding: This work has been carried out in the framework of the 5G-SOLUTIONS project, which has received funding from the European Union's Horizon 2020 research and innovation programme under grant agreement No 856691. The content of this paper reflects only the author's view; the EU Commission/Agency is not responsible for any use that may be made of the information it contains.

Institutional Review Board Statement: Not applicable.

Informed Consent Statement: Not applicable.

Data Availability Statement: Not applicable.

Acknowledgments: The authors gratefully acknowledge the 5G-SOLUTIONS consortium and the colleagues from the Consortium for the Research in Automation and Telecommunication (CRAT, https:/ / www.crat.eu/, accessed on 16 November 2021).

Conflicts of Interest: The authors declare no conflicts of interest. The funders had no role in the design of the study; in the collection, analyses, or interpretation of data; in the writing of the manuscript, or in the decision to publish the results.
Abbreviations
$\mathrm{CPO}$ Charging point operator
DSO Distribution system operator
PEV Plug-in electric vehicles
SOC State of charge

The following abbreviations are used in this manuscript:

\section{References}

1. Wang, Z.; Wu, W. Coordinated Control Method for DFIG-Based Wind Farm to Provide Primary Frequency Regulation Service. IEEE Trans. Power Syst. 2018, 33, 2644-2659. [CrossRef]

2. Buckspan, A.; Aho, J.; Fleming, P.; Jeong, Y.; Pao, L. Combining droop curve concepts with control systems for wind turbine active power control. In Proceedings of the 2012 IEEE Power Electronics and Machines in Wind Applications, Denver, CO, USA, 16-18 July 2012; pp. 1-8. [CrossRef]

3. Jietan, Z.; Linan, Q.; Pestana, R.; Fengkui, L.; Libin, Y. Dynamic frequency support by photovoltaic generation with "synthetic" inertia and frequency droop control. In Proceedings of the 2017 IEEE Conference on Energy Internet and Energy System Integration (EI2), Beijing, China, 26-28 November 2017; pp. 1-6. [CrossRef]

4. Lin, Y.; Barooah, P.; Meyn, S.; Middelkoop, T. Experimental Evaluation of Frequency Regulation From Commercial Building HVAC Systems. IEEE Trans. Smart Grid 2015, 6, 776-783. [CrossRef]

5. Hao, H.; Sanandaji, B.M.; Poolla, K.; Vincent, T.L. Frequency regulation from flexible loads: Potential, economics, and implementation. In Proceedings of the 2014 American Control Conference, Portland, OR, USA, 4-6 June 2014; pp. 65-72. [CrossRef]

6. Ko, K.; Sung, D.K. The Effect of Cellular Network-Based Communication Delays in an EV Aggregator's Domain on Frequency Regulation Service. IEEE Trans. Smart Grid 2019, 10, 65-73. [CrossRef]

7. Germanà, R.; De Santis, E.; Liberati, F.; Di Giorgio, A. On the Participation of Charging Point Operators to the Frequency Regulation Service using Plug-in Electric Vehicles and 5G Communications. In Proceedings of the 2021 IEEE International Conference on Environment and Electrical Engineering (EEEIC), Bari, Italy, 8-11 June 2021; pp. 1-6. [CrossRef]

8. Taleb, T.; Samdanis, K.; Mada, B.; Flinck, H.; Dutta, S.; Sabella, D. On Multi-Access Edge Computing: A Survey of the Emerging 5G Network Edge Cloud Architecture and Orchestration. IEEE Commun. Surv. Tutor. 2017, 19, 1657-1681. [CrossRef]

9. H2020 Project 5G-Solutions. 5G Solutions for European Citizens. Available online: https://5gsolutionsproject.eu/ (accessed on 29 July 2021). 
10. Yao, E.; Wong, V.W.S.; Schober, R. A robust design of electric vehicle frequency regulation service. In Proceedings of the 2014 IEEE International Conference on Smart Grid Communications (SmartGridComm), Venice, Italy, 3-6 November 2014 ; pp. 698-703. [CrossRef]

11. Yao, E.; Wong, V.W.S.; Schober, R. Optimization of Aggregate Capacity of PEVs for Frequency Regulation Service in Day-Ahead Market. IEEE Trans. Smart Grid 2018, 9, 3519-3529. [CrossRef]

12. Xia, S.; Bu, S.Q.; Luo, X.; Chan, K.W.; Lu, X. An Autonomous Real-Time Charging Strategy for Plug-In Electric Vehicles to Regulate Frequency of Distribution System With Fluctuating Wind Generation. IEEE Trans. Sustain. Energy 2018, 9, 511-524. [CrossRef]

13. Sanchez Gorostiza, F.; Gonzalez-Longatt, F. Optimised TSO-DSO interaction in unbalanced networks through frequencyresponsive EV clusters in virtual power plants. IET Gener. Transm. Distrib. 2020, 14, 4908-4917. [CrossRef]

14. Kuang, Y.; Li, C.; Zhou, B.; Cao, Y.; Yang, H.; Zeng, L. Asynchronous Method for Frequency Regulation by Dispersed Plug-in Electric Vehicles. Int. J. Emerg. Electr. Power Syst. 2018, 19, 20170158. [CrossRef]

15. Liu, H.; Qi, J.; Wang, J.; Li, P.; Li, C.; Wei, H. EV Dispatch Control for Supplementary Frequency Regulation Considering the Expectation of EV Owners. IEEE Trans. Smart Grid 2018, 9, 3763-3772. [CrossRef]

16. Jia, H.; Li, X.; Mu, Y.; Xu, C.; Jiang, Y.; Yu, X.; Wu, J.; Dong, C. Coordinated control for EV aggregators and power plants in frequency regulation considering time-varying delays. Appl. Energy 2018, 210, 1363-1376. [CrossRef]

17. Hashemi, S.; Arias, N.B.; Andersen, P.B.; Christensen, B.; Træholt, C. Frequency regulation provision using cross-brand bidirectional V2G-enabled electric vehicles. In Proceedings of the 2018 IEEE International Conference on Smart Energy Grid Engineering (SEGE), Oshawa, ON, Canada, 12-15 August 2018; pp. 249-254.

18. Cai, S.; Matsuhashi, R. Model Predictive Control for EV Aggregators Participating in System Frequency Regulation Market. IEEE Access 2021, 9, 80763-80771. [CrossRef]

19. Islam, M.M.; Zhong, X.; Sun, Z.; Xiong, H.; Hu, W. Real-time frequency regulation using aggregated electric vehicles in smart grid. Comput. Ind. Eng. 2019, 134, 11-26. [CrossRef]

20. Sbordone, D.A.; Carlini, E.M.; Di Pietra, B.; Devetsikiotis, M. The future interaction between virtual aggregator-TSO-DSO to increase DG penetration. In Proceedings of the 2015 International Conference on Smart Grid and Clean Energy Technologies (ICSGCE), Offenburg, Germany, 20-23 October 2015; pp. 201-205. [CrossRef]

21. Germanà, R.; Liberati, F.; Di Giorgio, A. Decentralized Model Predictive Control of Plug-in Electric Vehicles Charging based on the Alternating Direction Method of Multipliers. In Proceedings of the 2020 28th Mediterranean Conference on Control and Automation (MED), Saint-Raphaël, France, 15-18 September 2020; pp. 739-745. [CrossRef]

22. Di Giorgio, A.; Liberati, F.; Canale, S. Electric vehicles charging control in a smart grid: A model predictive control approach. Control Eng. Pract. 2014, 22, 147-162. [CrossRef]

23. TERNA S.p.A. Pilot Project Fast Reserve. Available online: https://www.terna.it/en/electric-system/pilot-projects-pursuantarera-resolution-300-2017-reel/fast-reserve-pilot-project (accessed on 29 July 2021).

24. Bezanson, J.; Edelman, A.; Karpinski, S.; Shah, V.B. Julia: A fresh approach to numerical computing. SIAM Rev. 2017, 59, 65-98. [CrossRef]

25. Gurobi Optimization, LLC. Gurobi Optimizer Reference Manual; 2021. Available online: https://www.gurobi.com/documentation/ 9.1/matlab_html/matlab_html.html (accessed on 16 November 2021). 